\title{
HIV infection and HERV expression: a review
}

\author{
Antoinette C van der Kuyl
}

\begin{abstract}
The human genome contains multiple copies of retrovirus genomes known as endogenous retroviruses (ERVs) that have entered the germ-line at some point in evolution. Several of these proviruses have retained (partial) coding capacity, so that a number of viral proteins or even virus particles are expressed under various conditions. Human ERVs (HERVs) belong to the beta-, gamma-, or spuma- retrovirus groups. Endogenous delta- and lenti- viruses are notably absent in humans, although endogenous lentivirus genomes have been found in lower primates.

Exogenous retroviruses that currently form a health threat to humans intriguingly belong to those absent groups. The best studied of the two infectious human retroviruses is the lentivirus human immunodeficiency virus (HIV) which has an overwhelming influence on its host by infecting cells of the immune system. One HIV-induced change is the induction of HERV transcription, often leading to induced HERV protein expression. This review will discuss the potential HIV-HERV interactions.

Several studies have suggested that HERV proteins are unlikely to complement defective HIV virions, nor is HIV able to package HERV transcripts, probably due to low levels of sequence similarity. It is unclear whether the expression of HERVs has a negative, neutral, or positive influence on HIV-AIDS disease progression. A positive effect was recently reported by the specific expression of HERVs in chronically HIV-infected patients, which results in the presentation of HERV-derived peptides to $\mathrm{CD} 8^{+}$T-cells. These cytotoxic T-cells were not tolerant to HERV peptides, as would be expected for self-antigens, and consequently lysed the HIV-infected, HERV-presenting cells. This novel mechanism could control HIV replication and result in a low plasma viral load. The possibility of developing a vaccination strategy based on these HERV peptides will be discussed.
\end{abstract}

\section{Review}

Retroviruses are unique among the viridae by inserting their genome into the host cellular DNA as an essential step in the viral replication cycle. Some older members of the retrovirus family have found their way into the vertebrate germ line while current members seem to remain exogenous. Vertebrate genomes contain substantial amounts of retroviral sequences in various states of inactivation since their integration (for a review on the discovery, see [1]). Integrated endogenous retrovirus (ERV) genomes commonly contain mutations, deletions, or are even reduced to single long terminal repeat (LTR) elements by homologous recombination between the two LTR's. More recent integrations usually have retained at least partial coding capacity. Some integrated ERV elements have been adopted and are used to the

Correspondence: a.c.vanderkuyl@amc.uva.nl

Laboratory of Experimental Virology, Department of Medical Microbiology, Center for Infection and Immunity Amsterdam (CINIMA), Academic Medical Center of the University of Amsterdam, Meibergdreef 15, 1105 AZ Amsterdam, The Netherlands hosts' advantage, either as a novel regulatory sequence, a novel protein, or as a means to protect against new retrovirus infections (reviewed by [2]). This latter mechanism is called superinfection resistance (SIR), and works best against closely related retroviruses by simple mechanisms such as receptor occupancy (reviewed by [3]).

Around $8 \%$ of the human genome is of retroviral origin, which includes proviruses that belong to beta-, gamma-, and spuma- retroviruses (Figure 1). Human endogenous retroviruses (HERVs) are historically classified by the tRNA specificity of their primer binding site (PBS), which can be confusing as unrelated species may share the same tRNA primer for reverse transcription [4]. Many HERV elements may have lost the ability to transfer, but several retain the capability to be transcribed and translated under certain conditions, including embryonic development and disease [2]. The most recent and widespread human integrations belong to retroviruses with homology to mouse mammary tumour virus (MMTV, a betaretrovirus) and are known as the 


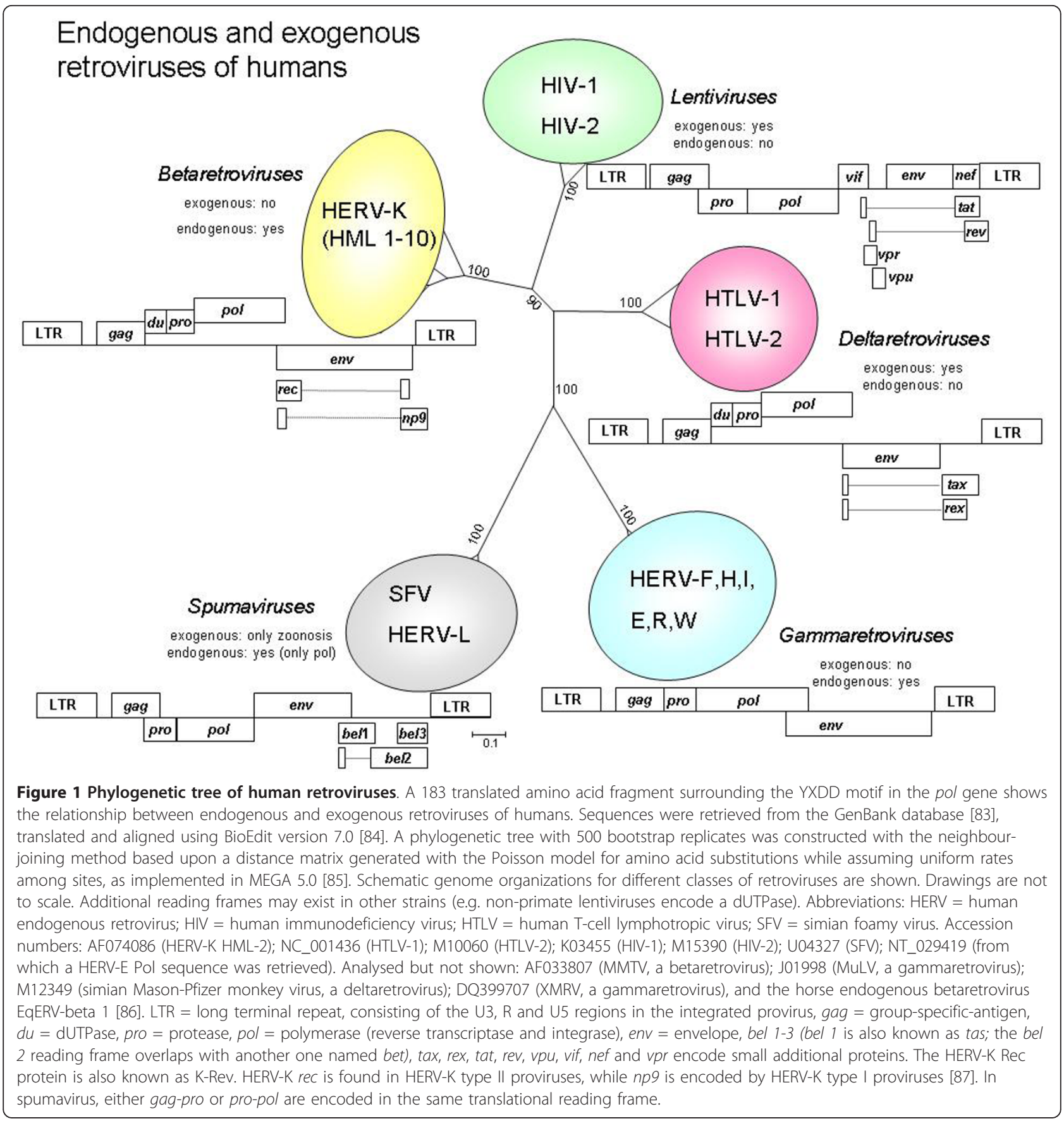

HERV-K (HML-2) (human MMTV-like) family (for a recent review, see [5]). Full-length proviral genomes of HML-2 family members are present, but these are not replication competent, even the HERV-K113 element that retains full coding capacity [6]. The human germ line tumour cell Tera- 1 even produces (non-infectious) retrovirus particles containing HML-2 RNA, but the assembly of these particles was found to depend on trans-acting viral proteins and RNA genomes derived from a mosaic of HML-2 proviral genomes [7]. The human mammary carcinoma cell line T74-D was found to release virions with B-type morphology that also contain retroviral transcripts originating from different loci [8]. Infectious HML-2 viruses have now been reconstructed in the laboratory to delineate their characteristics $[9,10]$.

Humans are currently threatened by only two exogenous retrovirus species: human $\mathrm{T}$-cell lymphotropic virus (HTLV, a deltaretrovirus) and human immunodeficiency virus (HIV, a lentivirus). A third putative human 
retrovirus, xenotropic murine leukaemia virus-related virus (XMRV, a gammaretrovirus) has recently been discarded as a contaminant $[11,12]$. No sequences with homology to delta- or lenti- viruses have been found in the human genome, although endogenous lentivirus genomes were discovered in basal primates $[13,14]$. Infection with the spumavirus simian foamy virus (SFV) occurs occasionally in persons exposed to non-human primates, but the virus does not seem to spread among humans [15-17]. The only described human endogenous retrovirus element with (distant) homology to foamy viruses is HERV-L [18].

In this review, the effect of infection with an exogenous retrovirus (HIV) on the resident endogenous virus population will be summarized with regard to viral genome expression, possible complementation, and immune response.

\section{Complementation of HERV and HIV proteins}

All retroviruses have a similar genetic make-up, whereby long terminal repeat (LTR) regulatory sequences flank the reading frames for Gag, Pol, and Env proteins. Gag and $\mathrm{Pol}$ are processed by the viral protease into smaller, functional proteins, while a cellular protease processes Env. The Pol polyprotein is cleaved into the protease (PR), reverse transcriptase (RT), and integrase (INT) enzymes. Homologous proteins encoded by one retrovirus could theoretically perform comparable functions in trans for another member of the family, and could thus complement a (defective) virus. Examples of possible complementation between HERV and HIV proteins will be discussed next.

A first example of a potential supplementary function is the dUTP pyrophophatase (dUTPase) enzyme. In betaretroviruses, a dUTPase domain is present at the Nterminal part of the protease. In non-primate exogenous lentiviruses, the dUTPase domain is located between the RNase $\mathrm{H}$ domain and the integrase coding region of the pol gene. Such dUTPase activity is essential for retrovirus replication by reducing the levels of dUTP during cDNA synthesis, thereby minimizing the incorporation of dUTP into the nascent DNA by the reverse transcriptase. In contrast to all other lentiviruses, exogenous primate lentiviruses (simian immunodefiency virus, SIV, and HIV) lack a dUTPase and depend upon the cellular uracil DNA-glycosylase UNG2 to provide this function [19]. Some researchers have suggested that HIV-1 can efficiently replicate without UNG2 [20], because it tolerates heavily uracilated DNA. Alternatively, the dUTPase encoded by active HERV-K proviruses could supply this function [21]. Interestingly, the endogenous lentivirus elements carried by lemurs do contain a dUTPase gene $[13,14]$, suggesting that this function was specifically lost in the extant SIV lineage.
When protease inhibitors specifically designed to inhibit the HIV-PR enzyme were included in the drug regimen offered to HIV-infected patients, there was some concern as to whether endogenous proteases, likely resistant to the anti-HIV drugs because of remote amino acid similarity, would be able to trans-complement the targeted enzyme. HERV-K10 protease is indeed highly resistant to HIV-PR inhibitors and able to process the HIV gag matrix-capsid protein at the authentic cleavage site [22]. However, when a HERV-K PR was incorporated into virions of a PR-defective HIV1 strain, no complementation was achieved [23]. The Gag-Pol precursors were found to be processed at unnatural sites, rendering the produced virion particles noninfectious. When HERV-K PR was incorporated into wild type (wt) HIV-1 virions with the intention to decrease their infectivity, no deleterious effect was seen, and only small fractions of aberrantly cleaved proteins were detected [23].

HERV-K10 INT has been reported to be able to complement an INT-defective HIV-1 strain, although infectivity was severely reduced to $3.7 \%$ of wt HIV-1 [24]. It is nevertheless remarkable that HERV-K10 INT was able to recognize the dissimilar HIV-1 LTR sequences to achieve in vitro integration levels of $10-30 \%$ of HIV-1 INT.

Retroviral envelope proteins are quite versatile and can often be used to package dissimilar viral genomes (pseudotyping). A few HERV envelope genes, such as the gammaretroviral HERV-W derived envelope protein, syncytin, that plays a role in the attachment of the human placenta, are expressed in vivo [25,26]. HERV-W Env can still function as a viral envelope protein, as infectious particles were generated when it was used to complement an env-defective HIV-1 strain [27]. The HERV-W pseudotyped particles were infectious for CD4-negative cells, suggesting a mechanism by which HIV-1 can expand its cellular tropism during natural infection. Interestingly, env-deficient HIV-1 cannot be complemented by the Env protein of murine leukaemia virus (MuLV), also a gammaretrovirus [26,27]. In contrast, the generation of pseudotypes between HIV-1 and endogenous xenotropic MuLVs was proposed earlier as an explanation for the increased tropism of HIV-1 particles harvested from a cell line that had been passaged in immunosuppressed mice [28].

Some classes of retroviruses, termed complex retroviruses, encode several accessory proteins that simple retroviruses lack. For instance, HIV-1 encodes at least 6 additional proteins, betaretroviruses generally only one, and gammaretroviruses commonly none. An essential additional protein that assists in transporting unspliced and incompletely spliced viral mRNAs from the nucleus to the cytoplasm is named Rev in HIV-1 and Rex in 
HTLV. The trans-acting Rev protein binds to a viral RNA structure in Env-encoding sequences that is denoted the Rev responsive element (RRE). A homolog of Rev/Rex is encoded by HERV-K, where it is termed $\mathrm{K}-\operatorname{Rev}$ or Rec [29]. To some extent, Rev/Rex proteins from complex retroviruses can complement each other, e.g. the Rex protein of HTLV-1 can functionally replace the HIV-1 Rev protein [30]. However, Rev/Rex complementation studies are not always straightforward because results often depend upon the experimental system used (see e.g. [31] and references therein). HIV-1 Rev can bind the HERV-K RRE, but the reverse is not true as K-Rev does not interact with the HIV-1 RRE [29]. Thus, although no actual complementation studies between HIV-Rev and K-Rev have been performed, it is unlikely that K-Rev can complement HIV-1 Rev because RRE binding is essential for protein function. Patients harbouring viral genomes with a rev defect or attenuating mutations have been described [32-34]. The disease phenotype in these patients is generally mild, suggesting that Rev defects in vivo are indeed not (completely) resolved by endogenous K-Rev.

\section{No HERV RNA in HIV-1 virions?}

Retrovirus particles contain, apart from two copies of the viral RNA genome, spliced viral RNA's, several tRNAs, and varying amounts of cellular mRNAs that are co-packaged in a concentration dependent manner [35-37]. Some of the cellular RNAs, such as those coding for ribosomal proteins, are preferentially packaged in HIV virions [35]. If HERV RNA would be co-packaged into HIV-1 virions, this could result in effective mobilization of these elements. This was investigated in several packaging cell lines used in retroviral vector systems. Zeilfelder et al. showed that HIV-1 derived vectors systems incorporate very little HERV transcripts in particles in contrast to murine leukaemia virus (MLV) based vectors [38]. Although the system used by Zeilfelder et al. is highly artificial, it nevertheless suggests that HIV-1 does not readily encapsidate HERV transcripts or genomes. An explanation could be that HIV-1 Gag does not recognize the RNA packaging signals carried by the non-lentiviral HERVs, while MLV Gag is able to bind to the more related HERV sequences.

\section{Increase of HERV viral RNA after HIV-1 infection}

In vitro $\mathrm{HIV}-1$ infection of either CD4 expressing $\mathrm{T}$ - cell lines or stimulated peripheral blood mononuclear cells (PBMC's) upregulates the production of HERV-K RNA and proteins compared to non-infected cells [39]. Analysis of blood plasma samples from HIV-1 infected patients also showed a significant increase of HERV-K RNA expression $[40,41]$, with titers as high as $10^{6}-10^{10}$ copies/ml [42]. In > 95\% of samples HERV-K pol RNA was found compared to only $5-8 \%$ of the control samples, which included hepatitis $\mathrm{C}$ virus (HCV) infected patients and healthy individuals [40]. In contrast, HERV-H RNA, which is upregulated in rheumatoid arthritis patients, was not detected in HIV-1 infected blood plasma [40]. The majority of the HERV transcripts in HIV-1 infected samples belonged to the HERV-K (HML-2) family and a minority to the HERVK (HML-3) cluster. These HERV-K transcripts were derived from several genomic loci, but transcripts from the HML-2 type-2 provirus located at chromosome 4 q35 predominated [42]. HML-2 type 1 and type 2 proviruses differ from each other by a 292 nt deletion in the env gene that disables the expression of functional protein in type 1 . Further analysis suggested that the observed transcripts likely represent full-length viral RNAs that are protected from degradation by Gag protein [40]. Indeed, immature virus particles that reacted only with anti-Gag Ig were observed using electron microscopy in a follow-up study, although mature viral particles reacting with anti-HERV-K Env antibodies and possessing condensed cores and symmetrically distributed spikes were also occasionally present in the blood plasma of HIV-1 infected patients [42]. HERV-K recombinant genomes were detected in blood plasma from some HIV-1 infected patients but not in breast cancer patients. Furthermore, genetic variation was observed in env (primarily synonymous substitutions indicative of purifying selection), with preservation of glycosylation sites together suggestive of replication through reverse transcription [42].

Laderoute et al. focussed on a HERV-K102 member of the HML-2 family that is upregulated during HIV-1 infection [43]. They reported that $\sim 76 \%$ of blood plasma samples of HIV-1 infected patients test positive for particle associated HERV-K102 pol transcripts, versus $\sim 3 \%$ of healthy controls. However, $\sim 79 \%$ and $\sim$ $62 \%$ of individuals with hepatitis B virus (HBV), hepatitis $\mathrm{C}$ virus $(\mathrm{HCV})$ or herpes viremia also showed increased particle associated HERV-K102 pol transcript expression [43], suggesting a more general mechanism of HERV-K upregulation due to virus infection and immune stimulation. HERV-K upregulation was independent of ethnicity as it was seen both in individuals of European and African descent [43].

In contrast, high throughput sequencing of transcripts from HIV-1 infected versus uninfected cells did not demonstrate a significant increase in HERV expression upon HIV-1 infection [44]. Possibly, the genetically highly aberrant SupT1 T-cell line used in these experiments can account for this unexpected result. Either HERV expression could already be at peak level before HIV infection of these cells, or specific HERV integrations could simply be absent. Alternatively, the vesicular 
stomatitis virus (VSV)-G envelope protein pseudo-typed HIV particles used could possibly explain the lack of HERV induction, as Contreras-Galindo et al. [39] used complete HIV-1 viral particles in their infection assays. Besides, virus infection conditions differed significantly between the experiments.

A significant upregulation of HERV-W and HERV-K RNA was detected in brain tissue from patients with AIDS dementia, whereas HERV-E expression was decreased [45]. The changes in HERV expression were associated with monocyte differentiation and macrophage activation.

\section{HERV expression during antiretroviral therapy}

As mentioned before, HERV-K RNA expression is increased in a dose-dependent manner by infection of peripheral blood mononuclear cells (PBMC's) and various cell lines with HIV-1 viral particles [39]. In vivo, it has not been examined whether HERV-K RNA levels increase during acute or chronic HIV-1 infection. Highly-active antiretroviral therapy (HAART) against HIV decreases the HIV plasma viral load ( $\mathrm{pVL}$ ) to $<50$ copies/ml in most patients. Quantifying the HERV-K RNA load in patients receiving HAART confirmed that the HERV-K load is linked to the HIV-1 pVL. Patients that showed a good suppression of HIV-1 replication had a HERV-K load that was around two orders of magnitude lower than the average HERV-K load in patients with less efficient HIV-1 suppression [46]. HERV-K102 particle-associated nucleic acid could not be measured in five patients on antiretroviral therapy (four of them with good control of the HIV-1 pVL) in another study, in contrast to patients not receiving antiretroviral therapy [43].

Longitudinal analysis of patients receiving HAART demonstrated the long-term suppression of the HERV-K $\mathrm{pVL}$ to $<5000$ copies/ml when the HIV-1 pVL remained below 50 copies $/ \mathrm{ml}$ [47]. When patients failed HAART, the HERV-K pVL increased rapidly, frequently preceding the HIV-1 pVL rebound [47]. It is unclear whether HERV-K is (partially) inhibited by the antiretroviral drugs, or whether the loss of activation by HIV-1, or possibly another mechanism, is responsible for the reduction in HERV-K load. Protease inhibitors targeted at HIV are not active against the HERV-K enzyme [22], but other classes of antiretroviral drugs, in particular the nucleotide analogues that target the RT enzyme, might be. It is, however, not likely that the HERV-encoded reverse transcriptases are appreciably involved in the increase in HERV load, as copy numbers of both intact $R T$ genes encoding functional proteins as well as intact primer binding sites (PBS) and other motifs needed to initiate reverse transcription, such as the primer activation domain (PAS) in HIV, are likely to be low. Also,
RT enzymes prefer certain tRNA molecules for initiation of replication and e.g. HIV-1 RT has decreased activity when using other tRNA/PBS combinations $[48,49]$. This preference suggests that RT activity on non-self templates is probably greatly reduced, disabling the reverse transcription of non-family members.

Interestingly, the non-nucleoside RT inhibitors nevirapine and efavirenz can efficiently inhibit endogenous RT activity that is detectable in many human cell lines and leukaemic cells, thereby attenuating the malignant phenotype of the cells $[50,51]$. Nevirapine was not active against LINE-1 retroposon-encoded RT, whereas several nucleoside analogue RT inhibitors did inhibit LINE-1 $\mathrm{RT}$, albeit with varying degrees of efficacy [52].

\section{Mechanisms of HERV activation by HIV infection}

It is not clear what the precise mechanism of HERV induction by HIV infection is. HERV expression is suppressed in the germ-line and during the first steps of embryogenesis, while it is controlled during cell development (reviewed in [53]). Increased HERV expression is often seen during carcinogenesis and in autoimmune diseases such as multiple sclerosis [2], but it is unclear whether the increased expression is a cause or consequence of the disease. HERVs including the HERV-K (HML-2) family [54] and HIV-1 proviruses [55] are normally transcriptionally silenced by $\mathrm{CpG}$ methylation that is subsequently guarded by DNA methyltransferase 1 (DNMT1) (reviewed in [53]). Loss of methylation results in activation of proviruses, either HERV or HIV, but it has not been investigated whether HIV actively influences proviral methylation levels. HERV expression in HIV-infected cells could be enhanced if methylation levels of proviral DNA are reduced by HIV. Other investigators proposed a more complex mechanism for the induction of HIV-1 latency that employs transcriptional interference $[56,57]$. Several mechanisms may cooperate to restrict HIV provirus activation [58]. It is not clear whether changes in the pool of available transcription factors modulates HERV expression. Many HERV LTR promoters are probably not fully functional, e.g. not able to bind these transcription factors, and thus do no longer require such a repressive mechanism. Additionally, the HIV-1 proviral integration site location might also select for viral latency, virus induction or stable expression [59]. Generally, HIV-1 favours integration into transcriptionally active genes, usually in introns that are part of local transcription hotspots, without apparent directional bias [60]. In contrast, HERV proviruses are generally found outside genes, and commonly in the reverse orientation compared to the nearby cellular gene. HERV integrations seen in contemporary genomes are the result of stringent evolutionary selection, whereby HERV's in the sense orientation 
(facilitates transcription with nearby genes) or within cellular genes were probably negatively selected for [60]. In other words, observed HERV integrations do probably not faithfully reflect HERV target site preferences. Anyhow, the consequence is that HIV-1 proviruses are rarely found in the proximity of HERV integrations $[59,60]$, ruling out that there is a direct positional effect of HIV-1 transcription upon HERV activation.

Specific upregulation of endogenous proviruses by HIV proteins or cellular proteins upregulated by HIV is another possibility. It has been suggested that the HIV-1 Tat protein is able to directly activate the HERV-K (HML-2) LTR [47]. The HTLV-I encoded transactivator protein Tax is likewise able to activate HERV LTRs, mainly of HERV-W and HERV-H [61]. HERV-K (HML2) RNA is not, or only weakly, upregulated by common stimulation of donor PBMCs with phytohaemagglutinin, gamma irradiation or 5-azacytidine [62], suggesting that HERV upregulation requires undefined, yet specific conditions.

Another indirect mechanism of HERV upregulation by HIV-1 infection could be through opportunistic viral infections. HIV infection deregulates and eventually destroys the immune system. Loss of immune control facilitates the replication of diverse opportunistic pathogens. Some of these pathogens, e.g. herpesviruses, are virtually ubiquitous in the human population and remain latently present during the lifetime of the host. Herpesviruses are reactivated from latency during chronic HIV infection [63]. Herpes simplex virus type 1 (HSV-1) can specifically activate the HERV-W LTR, and induce expression of HERV-W Gag and Env proteins in cell lines [64-66]. Epstein-Barr virus (EBV), that has a prevalence rate of around $75 \%$ in humans, induces transcription of the $e n v$ gene of HERV-K18 [67], and so do human herpesvirus 6A [68] and 6B [69]. Most likely, the other five human herpesviruses are also able to activate HERV transcription. HHV-6 and human herpesvirus 7 (HHV-7), that have infection rates of $100 \%$ each in the human population, infect, similar to $\mathrm{HIV}, \mathrm{CD} 4^{+} \mathrm{T}$-cells, and could thus (partly) be responsible for HERV induction seen in PBMC's after HIV-1 infection. EBV commonly infects B-cells, while cytomegalovirus (CMV), with a prevalence rate of around 50\%, infects monocytes and macrophages, and both could likewise contribute to the observed rise in PBMC HERV expression. In addition, infection by influenza A virus enhanced HERV-W expression in cell culture as well [66], suggesting that many more viruses have the potential to do so.

Expression of the HIV accessory protein Vif counteracts the viral restriction factor APOBEC3G and could result in abrogation of cellular HERV control [70] and de novo replication of HERV elements by reverse transcription. Restriction during reverse transcription by
APOBEC3G was shown for HERV-K (HML-2) elements, both during in vitro replication as well as in the mutation pattern of ancient integrations [71,72].

\section{HERV proteins and antibodies in HIV infected patients}

Increased expression of HERV-K Gag protein has been detected in both the $\mathrm{CD}^{+}$and $\mathrm{CD}^{+} \mathrm{T}$-cell fractions from HIV-infected patients compared to T-cells from uninfected individuals [39]. It is remarkable that HERV$\mathrm{K}$ Gag is also upregulated in $\mathrm{CD}^{+} \mathrm{T}$-cells that are normally not infected by HIV-1, which would suggest a more general and indirect mechanism of activation. No significant correlation was found between the HIV-1 pVL, $\mathrm{CD}^{+}$or $\mathrm{CD}^{+}{ }^{+} \mathrm{T}$-cell counts and HERV-K protein titers.

Antibodies to expressed HERV-K proteins are frequently found in several groups of patients, which is interesting because HERVs should be classified as selfantigens. Some studies did describe a HERV-K antibody response in $\mathrm{HIV}-1$ infected persons, while others did not. No significant increase in serum antibodies against recombinant human teratocarcinoma-derived virus (HTDV)-HERV-K Env protein was seen in HIV-1 infected patients ( $15.2 \%$ versus $12.6 \%$ in healthy controls) [73]. Similar negative results were obtained by Vogetseder et al. [73] and Boller et al. [74], when examining the antibody response against HTDV/HERV-K Gag and Env proteins in HIV-1 positive individuals, and by Goedert et al. [75] in HIV-1 infected individuals with testicular cancer. In contrast, $70 \%$ of HIV-1 infected patients versus $3 \%$ of healthy controls tested positive for antibodies against HTDV/HERV-K Env in a fourth study [76]. A fifth study, employing HERV-K102 Env peptide serology reported similar numbers, with $70-80 \%$ of $\mathrm{HIV}-1$ viraemic patients testing positive versus $2 \%$ of healthy controls and $18 \%$ of patients with herpes viraemia [43]. Interestingly, a patient seroconverting for antiHIV-1 antibodies showed a parallel seroconversion for anti-HERV-K Env antibodies in the study by Vogetseder et al. [73]. Cross-reactivity is unlikely to explain this result, as the HERV-K and HIV-1 Env proteins have little amino acid similarity.

Testing urine specimens of HIV-1 infected individuals and healthy controls for antibodies against a peptide derived from the gammaretrovirus HERV-E Env protein showed that $36.4 \%$ and $81.3 \%$ of patients in the CDC AIDS categories $\mathrm{A}$ and $\mathrm{B} / \mathrm{C}$ respectively, contained specific antibodies, while none of the HIV-negative controls tested positive [77]. Fourteen peptides derived from various endogenous retroviruses were used to search for IgM and IgG antibodies in HIV-1 infected and uninfected immunosuppressed persons by Lawoko et al. [78]. Three peptides, corresponding to the C-terminal 
half of the murine leukaemia virus capsid protein, a conserved domain of the HERV-H transmembrane domain, and part of HERV-K HML-3 encoded Pol, were found to bind IgG in both groups, with stronger binding of the latter two peptides in HIV-1 infected patients [78]. No correlation of either IgM or IgG binding with progression to AIDS was observed.

In conclusion, it is difficult to summarize these studies on anti-HERV antibodies in HIV-1 infected patients. Studies on HERV-K remain contradictory, while others investigated HERV families whose RNA expression levels during HIV infection have not been characterized.

\section{T-cell response to HERV in HIV infected patients}

Although HERV proteins should be regarded as selfantigens, tolerance is often broken during neoplastic processes and also during HIV infection as demonstrated by a study that measured $\mathrm{T}$-cell responses against HERV during HIV infection [41]. Peptides corresponding to HERV and HIV epitopes were tested for their ability to evoke a T-cell response in vitro. Ten peptides contained short regions of homology between HERV-H/-K/-L and HIV, respectively, and six others unique for HERV-L or HERV-W were used to estimate both putative cross-reactive and virus-specific T-cell responses. HERV-H and HERV-W cluster with the gammaretroviruses, and HERV-L has similarity to spumaviruses [79]. PBMC samples were collected from HIV-1 infected, HCV infected, and uninfected individuals. Tcell responses against HERV epitopes were detected only in HIV-1 positive patients, with no significant difference in reactions against unique HERV peptides versus HIV-related HERV peptides. This suggests that the response against HERV epitopes is distinct from the anti-HIV response. T-cell cross-reactivity was tested in three patients with a response to either the HIV-RT VL9 or HERV-L II9 peptide which share some amino acid similarity, but was found to be absent.

Anti-HERV T cell responses were broad and varied significantly among the study subjects [41]. The HERVspecific $\mathrm{T}$-cell responses were inversely correlated with the HIV-1 pVL, raising the exciting possibility that these responses could be involved in the control of HIV replication. Reactivity to the unique HERV-L IQ10 peptide was found in 5/16 HIV-1 infected patients. Longitudinal analysis of three patients showed persistent responses to this peptide that declined in two patients when HAART was started. The third patient with the highest response to this peptide controlled HIV replication without therapy. HERV-specific CD8 ${ }^{+}$T-cells were able to lyse cells presenting the corresponding peptide. If HIV-1 infected cells that present HERV peptides are subsequently lysed by HERV-specific $\mathrm{CD} 8^{+}$T-cells, a decrease in HIV-1 pVL would be the result, explaining the observations in the three patients described above. In a follow-up study, anti-HERV T-cell responses were analysed in a larger cohort of untreated HIV-1 infected individuals [70]. The breadth and magnitude of the HERV response were again inversely correlated with HIV-1 pVL and positively associated with $\mathrm{CD} 4^{+} \mathrm{T}$-cell counts. The peptide that evoked the largest number of responses was derived from HERV-H Env. Other peptides tested originated from HERV-K $(\mathrm{n}=14)$, HERV-L $(\mathrm{n}=12)$, and HERV$\mathrm{W}(\mathrm{n}=1)$.

An HIV-1 positive patient who controlled the infection for over 8 years showed HLA-B51 restricted responses to the HERV-K Pol epitope FAFTIPAI as well as to the corresponding HIV-1 epitope TAFTIPSI. The $\mathrm{CD} 8^{+} \mathrm{T}$-cells responding to the HERV epitope were found to be less activated and more differentiated than the cells targeting the HIV-1 epitope [70]. The cytomegalovirus-specific $\mathrm{CD} 8^{+} \mathrm{T}$-cell population in this patient did mimic the HERV response.

Overall, these results suggest that a robust $\mathrm{CD} 8^{+} \mathrm{T}$ cell response against the newly expressed HERV peptides presented by HIV infected cells may contribute to the control of HIV replication in blood. Indeed, a second study in vertically infected children showed a similar outcome: anti-HERV responses were inversely correlated with the HIV-1 plasma viral load and positively correlated with the $\mathrm{CD} 4^{+} \mathrm{T}$ cell count [80]. Alternatively, Tandon et al. suggest that instead of a direct effect of anti-HERV responses, the correlations might be related to a more intact immune system in patients showing considerable anti-HERV activity.

\section{Anti-HERV vaccines as an approach to combat HIV?}

T-cell responses that are effective in lowering the HIV-1 $\mathrm{pVL}$ and thus assist in controlling HIV replication are potential vaccine targets. In contrast to the replicating and rapidly mutating HIV-1 genome, HERVS are cellular genes that are not prone to mutation. Although HERV integrations are present in every cell in the body, they are expressed in a cell type and development specific way, making many HERV proteins rather specific antigens. Indeed, a HERV-K env transcript, HERV-KMEL, encoded a melanoma-specific antigen that was recognized by cytolytic $\mathrm{T}$ lymphocytes [81]. Vaccination with peptides derived from another melanoma-specific antigen had been successful earlier in inducing tumour regression (see [81]), suggesting that vaccination with HERV-K-MEL peptides could be an effective strategy. A HERV-E transcript specifically expressed by renal cell carcinoma tissue and cell lines encoded a peptide that was recognized by $\mathrm{CD} 8^{+} \mathrm{T}$-cells after allogeneic hematopoietic stem cell transplantation [82]. Recognition resulted in tumour regression in vivo, an example of the 
therapeutic power of targeting HERV epitopes in malignancy.

The research performed by Garrison et al. [41], SenGupta et al. [70], and Tandon et al. [80] suggests that a similar approach is possible in HIV-1 infection. T-cell responses targeted at HERVs expressed by HIV-1 infected cells could be helpful in killing those cells. However, before embarking on the HERV approach, several basic issues need to be addressed. It is currently unclear which HERV epitopes are expressed by HIVinfected cells, at what magnitude and at what specificity. SenGupta et al. recorded $\mathrm{CD} 8^{+} \mathrm{T}$-cell responses against multiple HERV families in HIV-1 infected patients, including HERV-H, - K, - L, and -W [70]. HERV epitope recognition was found to vary among individuals, complicating the generation of a universal vaccine. It is also uncertain whether the discovered epitopes are expressed exclusively by HIV-1 infected cells, or whether there is also an indirect bystander effect on non-infected cells as reported by Contreras-Galindo et al. [39]. Furthermore, it is not clear if a similar pattern of HERV expression is apparent in all HIV-1 susceptible cell types e.g. in $\mathrm{CD} 4^{+}$ $\mathrm{T}$-cells and monocytes. These issues need to be addressed in vitro and combined with the analysis of patient samples before HERV-vaccination approaches can be developed further.

\section{Conclusions}

Infection of humans with the retrovirus HIV-1 has profound effects upon the resident, endogenous retroviruses. Transcripts and proteins representing diverse classes of endogenous retroviruses are upregulated. Direct interactions between HERVs and HIV-1 are limited as indicated by the lack of complementation, which probably relates to the low sequence similarity. Induction of certain HERV epitopes on HIV-1 infected cells renders these cells susceptible to lysis by $\mathrm{CD} 8^{+} \mathrm{T}$-cells, constituting a possible mechanism to control HIV-1 infection. It has been proposed to translate these observations into a vaccination approach with stable HERVbased antigens, but the detailed knowledge on the magnitude and specificity of HERV expression in HIV-1 infected cells is prerequisite for such applications.

\footnotetext{
Acknowledgements

The author thanks Ben Berkhout and Marion Cornelissen for critical reading of the manuscript.
}

\section{Authors' contributions}

ACvdK conceived the review topic and drafted the manuscript.

\section{Competing interests}

The author declares that she has no competing interests.

Received: 9 December 2011 Accepted: 16 January 2012 Published: 16 January 2012
References

1. Weiss RA: The discovery of endogenous retroviruses. Retrovirology 2006, 3:67.

2. Kurth $R$, Bannert N: Beneficial and detrimental effects of human endogenous retroviruses. Int J Cancer 2010, 126:306-314.

3. Nethe M, Berkhout B, van der Kuyl AC: Retroviral superinfection resistance. Retrovirology 2005, 2:52.

4. Blomberg J, Benachenhou F, Blikstad V, Sperber G, Mayer J: Classification and nomenclature of endogenous retroviral sequences (ERVs): problems and recommendations. Gene 2009, 448:115-123.

5. Mayer J, Meese E: Human endogenous retroviruses in the primate lineage and their influence on host genomes. Cytogenet Genome Res 2005, 110:448-456.

6. Beimforde N, Hanke K, Ammar I, Kurth R, Bannert N: Molecular cloning and functional characterization of the human endogenous retrovirus K113. Virology 2008, 371:216-225.

7. Ruprecht K, Ferreira H, Flockerzi A, Wahl S, Sauter M, Mayer J, et al: Human endogenous retrovirus family HERV-K(HML-2) RNA transcripts are selectively packaged into retroviral particles produced by the human germ cell tumor line Tera- 1 and originate mainly from a provirus on chromosome 22q11.21. J Virol 2008, 82:10008-10016.

8. Seifarth W, Skladny H, Krieg-Schneider F, Reichert A, Hehlmann R, LeibMosch C: Retrovirus-like particles released from the human breast cancer cell line T47-D display type B- and C-related endogenous retroviral sequences. J Virol 1995, 69:6408-6416.

9. Dewannieux M, Harper F, Richaud A, Letzelter C, Ribet D, Pierron G, et al: Identification of an infectious progenitor for the multiple-copy HERV-K human endogenous retroelements. Genome Res 2006, 16:1548-1556.

10. Lee YN, Bieniasz PD: Reconstitution of an infectious human endogenous retrovirus. PLoS Pathog 2007, 3:e10

11. van der Kuyl AC, Cornelissen M, Berkhout B: Of mice and men: on the origin of XMRV. Frontiers in Microbiology 2010, 1:147.

12. Paprotka T, Delviks-Frankenberry KA, Cingoz O, Martinez A, Kung HJ, Tepper CG, et al: Recombinant origin of the retrovirus XMRV. Science 2011, 333:97-101.

13. Gifford RJ, Katzourakis A, Tristem M, Pybus OG, Winters M, Shafer RW: A transitional endogenous lentivirus from the genome of a basal primate and implications for lentivirus evolution. Proc Natl Acad Sci USA 2008, 105:20362-20367.

14. Gilbert C, Maxfield DG, Goodman SM, Feschotte C: Parallel germline infiltration of a lentivirus in two Malagasy lemurs. PLoS Genet 2009, 5: e1000425.

15. Switzer WM, Bhullar V, Shanmugam V, Cong ME, Parekh B, Lerche NW, et al: Frequent simian foamy virus infection in persons occupationally exposed to nonhuman primates. J Virol 2004, 78:2780-2789.

16. Calattini S, Betsem EB, Froment A, Mauclere P, Tortevoye P, Schmitt C, et al: Simian foamy virus transmission from apes to humans, rural Cameroon. Emerg Infect Dis 2007, 13:1314-1320.

17. Jones-Engel L, May CC, Engel GA, Steinkraus KA, Schillaci MA, Fuentes A, et al: Diverse contexts of zoonotic transmission of simian foamy viruses in Asia. Emerg Infect Dis 2008, 14:1200-1208.

18. Cordonnier A, Casella JF, Heidmann T: Isolation of novel human endogenous retrovirus-like elements with foamy virus-related pol sequence. J Virol 1995, 69:5890-5897.

19. Priet S, Gros N, Navarro JM, Boretto J, Canard B, Querat G, et al: HIV-1associated uracil DNA glycosylase activity controls dUTP misincorporation in viral DNA and is essential to the HIV-1 life cycle. Mol Cell 2005, 17:479-490.

20. Yan N, O'Day E, Wheeler LA, Engelman A, Lieberman J: HIV DNA is heavily uracilated, which protects it from autointegration. Proc Natl Acad Sci USA 2011, 108:9244-9249.

21. McIntosh EM, Haynes RH: HIV and human endogenous retroviruses: an hypothesis with therapeutic implications. Acta Biochim Pol 1996, 43:583-592.

22. Towler EM, Gulnik SV, Bhat TN, Xie D, Gustschina E, Sumpter TR, et al: Functional characterization of the protease of human endogenous retrovirus, K10: can it complement HIV-1 protease? Biochemistry 1998, 37:17137-17144.

23. Padow M, Lai L, Fisher RJ, Zhou YC, Wu X, Kappes JC, et al: Analysis of human immunodeficiency virus type 1 containing HERV-K protease. AIDS Res Hum Retroviruses 2000, 16:1973-1980. 
24. Ogata T, Okui N, Sakuma R, Kobayashi N, Kitamura Y: Integrase of human endogenous retrovirus $\mathrm{K}-10$ supports the replication of replicationincompetent Int- human immunodeficiency virus type 1 mutant. Jpn J Infect Dis 1999, 52:251-252.

25. Mi S, Lee X, Li X, Veldman GM, Finnerty H, Racie L, et al: Syncytin is a captive retroviral envelope protein involved in human placental morphogenesis. Nature 2000, 403:785-789.

26. Blond $J$, Lavillette D, Cheynet V, Bouton O, Oriol G, Chapel-Fernandes S, et al: An envelope glycoprotein of the human endogenous retrovirus HERV-W is expressed in the human placenta and fuses cells expressing the type D mammalian retrovirus receptor. J Virol 2000, 74:3321-3329.

27. An DS, Xie Y, Chen IS: Envelope gene of the human endogenous retrovirus HERV-W encodes a functional retrovirus envelope. J Virol 2001, 75:3488-3489

28. Lusso P, di M, Ensoli B, Franchini G, Jemma C, DeRocco SE, et al: Expanded HIV-1 cellular tropism by phenotypic mixing with murine endogenous retroviruses. Science 1990, 247:848-852.

29. Yang J, Bogerd HP, Peng S, Wiegand H, Truant R, Cullen BR: An ancient family of human endogenous retroviruses encodes a functional homolog of the HIV-1 Rev protein. Proc Natl Acad Sci USA 1999, 96:13404-13408.

30. Rimsky L, Hauber J, Dukovich M, Malim MH, Langlois A, Cullen BR, et al: Functional replacement of the HIV-1 rev protein by the HTLV-1 rex protein. Nature 1988, 335:738-740.

31. Sakai H, Siomi H, Shida H, Shibata R, Kiyomasu T, Adachi A: Functional comparison of transactivation by human retrovirus rev and rex genes. J Virol 1990, 64:5833-5839.

32. Iversen AK, Shpaer EG, Rodrigo AG, Hirsch MS, Walker BD, Sheppard HW et al: Persistence of attenuated rev genes in a human immunodeficiency virus type 1-infected asymptomatic individual. J Virol 1995, 69:5743-5753.

33. Saurya S, Lichtenstein Z, Karpas A: Defective rev response element (RRE) and rev gene in HAART treated AIDS patients with discordance between viral load and CD4+ T-cell counts. J Clin Virol 2005, 33:324-327.

34. Churchill MJ, Chiavaroli L, Wesselingh SL, Gorry PR: Persistence of attenuated HIV-1 rev alleles in an epidemiologically linked cohort of long-term survivors infected with nef-deleted virus. Retrovirology 2007 4:43.

35. Rulli SJ, Jr. Hibbert CS, Mirro J, Pederson T, Biswal S, Rein A: Selective and nonselective packaging of cellular RNAs in retrovirus particles. J Virol 2007, 81:6623-6631.

36. Houzet L, Paillart JC, Smagulova F, Maurel S, Morichaud Z, Marquet R, et al: HIV controls the selective packaging of genomic, spliced viral and cellular RNAs into virions through different mechanisms. Nucleic Acids Res 2007, 35:2695-2704.

37. Didierlaurent L, Racine PJ, Houzet L, Chamontin C, Berkhout B, Mougel M: Role of HIV-1 RNA and protein determinants for the selective packaging of spliced and unspliced viral RNA and host U6 and 7SL RNA in virus particles. Nucleic Acids Res 2011, 39:8915-8927.

38. Zeilfelder U, Frank O, Sparacio S, Schon U, Bosch V, Seifarth W, et al: The potential of retroviral vectors to cotransfer human endogenous retroviruses (HERVs) from human packaging cell lines. Gene 2007, 390:175-179.

39. Contreras-Galindo R, Lopez P, Velez R, Yamamura Y: HIV-1 infection increases the expression of human endogenous retroviruses type $\mathrm{K}$ (HERV-K) in vitro. AIDS Res Hum Retroviruses 2007, 23:116-122.

40. Contreras-Galindo R, Kaplan MH, Markovitz DM, Lorenzo E, Yamamura Y: Detection of HERV-K(HML-2) viral RNA in plasma of HIV type 1-infected individuals. AIDS Res Hum Retroviruses 2006, 22:979-984.

41. Garrison KE, Jones RB, Meiklejohn DA, Anwar N, Ndhlovu LC, Chapman JM, et al: T cell responses to human endogenous retroviruses in HIV-1 infection. PLoS Pathog 2007, 3:e165.

42. Contreras-Galindo RA, Kaplan MH, Contreras-Galindo AC, GonzalezHernandez M, Ferlengui I, Giusti F, et al: Characterization of human endogenous retroviral elements in the blood of HIV-1-infected individuals. J Virol 2012, 86(1):262-76.

43. Laderoute MP, Giulivi A, Larocque L, Bellfoy D, Hou Y, Wu HX, et al: The replicative activity of human endogenous retrovirus K102 (HERV-K102) with HIV viremia. AIDS 2007, 21:2417-2424.

44. Lefebvre G, Desfarges S, Uyttebroeck F, Munoz M, Beerenwinkel N, Rougemont J, et al: Analysis of HIV-1 expression level and sense of transcription by high-throughput sequencing of the infected cell. $J$ Virol 2011, 85:6205-6211.

45. Johnston JB, Silva C, Holden J, Warren KG, Clark AW, Power C: Monocyte activation and differentiation augment human endogenous retrovirus expression: implications for inflammatory brain diseases. Ann Neurol 2001, 50:434-442.

46. Contreras-Galindo R, Gonzalez M, Almodovar-Camacho S, GonzalezRamirez S, Lorenzo E, Yamamura Y: A new real-time-RT-PCR for quantitation of human endogenous retroviruses type K (HERV-K) RNA load in plasma samples: increased HERV-K RNA titers in HIV-1 patients with HAART non-suppressive regimens. J Virol Methods 2006, 136:51-57.

47. Contreras-Galindo R, Almodovar-Camacho S, Gonzalez-Ramirez S, Lorenzo E, Yamamura Y: Comparative longitudinal studies of HERV-K and HIV-1 RNA titers in HIV-1-infected patients receiving successful versus unsuccessful highly active antiretroviral therapy. AIDS Res Hum Retroviruses 2007, 23:1083-1086.

48. Oude Essink BB, Das AT, Berkhout B: HIV-1 reverse transcriptase discriminates against non-self tRNA primers. J Mol Biol 1996, 264:243-254.

49. Abbink TE, Beerens $N$, Berkhout B: Forced selection of a human immunodeficiency virus type 1 variant that uses a non-self tRNA primer for reverse transcription: involvement of viral RNA sequences and the reverse transcriptase enzyme. J Virol 2004, 78:10706-10714.

50. Mangiacasale R, Pittoggi C, Sciamanna I, Careddu A, Mattei E, Lorenzini R, et al: Exposure of normal and transformed cells to nevirapine, a reverse transcriptase inhibitor, reduces cell growth and promotes differentiation. Oncogene 2003, 22:2750-2761.

51. Sciamanna I, Landriscina M, Pittoggi C, Quirino M, Mearelli C, Beraldi R, et al: Inhibition of endogenous reverse transcriptase antagonizes human tumor growth. Oncogene 2005, 24:3923-3931.

52. Jones RB, Garrison KE, Wong JC, Duan EH, Nixon DF, Ostrowski MA: Nucleoside analogue reverse transcriptase inhibitors differentially inhibit human LINE-1 retrotransposition. PLOS ONE 2008, 3:e1547.

53. Rowe HM, Trono D: Dynamic control of endogenous retroviruses during development. Virology 2011, 411:273-287.

54. Lavie L, Kitova M, Maldener E, Meese E, Mayer J: CpG methylation directly regulates transcriptional activity of the human endogenous retrovirus family HERV-K(HML-2). J Virol 2005, 79:876-883.

55. Blazkova J, Trejbalova K, Gondois-Rey F, Halfon P, Philibert P, Guiguen A, et al: CpG methylation controls reactivation of HIV from latency. PLOS Pathog 2009, 5:e1000554.

56. Lenasi T, Contreras X, Peterlin BM: Transcriptional interference antagonizes proviral gene expression to promote HIV latency. Cell Host Microbe 2008, 4:123-133.

57. Duverger A, Jones J, May J, Bibollet-Ruche F, Wagner FA, Cron RQ, et al: Determinants of the establishment of human immunodeficiency virus type 1 latency. J Virol 2009, 83:3078-3093.

58. Richman DD, Margolis DM, Delaney M, Greene WC, Hazuda D, Pomerantz RJ: The challenge of finding a cure for HIV infection. Science 2009, 323:1304-1307.

59. Lewinski MK, Bisgrove D, Shinn P, Chen H, Hoffmann C, Hannenhalli S, et al: Genome-wide analysis of chromosomal features repressing human immunodeficiency virus transcription. J Virol 2005, 79:6610-6619.

60. Schroder AR, Shinn P, Chen H, Berry C, Ecker JR, Bushman F: HIV-1 integration in the human genome favors active genes and local hotspots. Cell 2002, 110:521-529.

61. Toufaily C, Landry S, Leib-Mosch C, Rassart E, Barbeau B: Activation of LTRs from different human endogenous retrovirus (HERV) families by the HTLV-1 Tax protein and T-cell activators. Viruses 2011, 3:2146-2159.

62. Depil S, Roche C, Dussart P, Prin L: Expression of a human endogenous retrovirus, HERV-K, in the blood cells of leukemia patients. Leukemia 2002, 16:254-259.

63. Griffiths PD: Herpesviruses and AIDS. J Antimicrob Chemother 1996, 37(Suppl B):87-95.

64. Lee WJ, Kwun HJ, Kim HS, Jang KL: Activation of the human endogenous retrovirus $\mathrm{W}$ long terminal repeat by herpes simplex virus type 1 immediate early protein 1. Mol Cells 2003, 15:75-80.

65. Ruprecht K, Obojes K, Wengel V, Gronen F, Kim KS, Perron H, et al: Regulation of human endogenous retrovirus $\mathrm{W}$ protein expression by herpes simplex virus type 1 : implications for multiple sclerosis. $J$ Neurovirol 2006, 12:65-71. 
66. Nellaker $\mathrm{C}$, Yao $Y$, Jones-Brando L, Mallet F, Yolken $\mathrm{RH}$, Karlsson $\mathrm{H}$ : Transactivation of elements in the human endogenous retrovirus $\mathrm{W}$ family by viral infection. Retrovirology 2006, 3:44.

67. Sutkowski N, Conrad B, Thorley-Lawson DA, Huber BT: Epstein-Barr virus transactivates the human endogenous retrovirus HERV-K18 that encodes a superantigen. Immunity 2001, 15:579-589.

68. Tai AK, Luka J, Ablashi D, Huber BT: HHV-6A infection induces expression of HERV-K18-encoded superantigen. J Clin Virol 2009, 46:47-48.

69. Turcanova VL, Bundgaard B, Hollsberg P: Human herpesvirus- $6 B$ induces expression of the human endogenous retrovirus K18-encoded superantigen. J Clin Virol 2009, 46:15-19.

70. SenGupta D, Tandon R, Vieira RG, Ndhlovu LC, Lown-Hecht R, Ormsby CE, et al: Strong human endogenous retrovirus-specific $T$ cell responses are associated with control of HIV-1 in chronic infection. J Virol 2011, 85:6977-6985.

71. Lee YN, Malim MH, Bieniasz PD: Hypermutation of an ancient human retrovirus by APOBEC3G. J Virol 2008, 82:8762-8770.

72. Armitage AE, Katzourakis A, de OT, Welch JJ, Belshaw R, Bishop KN, et al: Conserved footprints of APOBEC3G on hypermutated human immunodeficiency virus type 1 and human endogenous retrovirus HERV-K(HML2) sequences. J Virol 2008, 82:8743-8761.

73. Vogetseder W, Dumfahrt A, Mayersbach P, Schonitzer D, Dierich MP. Antibodies in human sera recognizing a recombinant outer membrane protein encoded by the envelope gene of the human endogenous retrovirus K. AIDS Res Hum Retroviruses 1993, 9:687-694.

74. Boller K, Janssen O, Schuldes H, Tonjes RR, Kurth R: Characterization of the antibody response specific for the human endogenous retrovirus HTDV/ HERV-K. J Virol 1997, 71:4581-4588.

75. Goedert JJ, Sauter ME, Jacobson LP, Vessella RL, Hilgartner MW, Leitman SF, et al: High prevalence of antibodies against HERV-K10 in patients with testicular cancer but not with AIDS. Cancer Epidemiol Biomarkers Prev 1999, 8:293-296.

76. Lower R, Lower J, Kurth R: The viruses in all of us: characteristics and biological significance of human endogenous retrovirus sequences. Proc Natl Acad Sci USA 1996, 93:5177-5184.

77. Stevens RW, Baltch AL, Smith RP, McCreedy BJ, Michelsen PB, Bopp LH, et al: Antibody to human endogenous retrovirus peptide in urine of human immunodeficiency virus type 1-positive patients. Clin Diagn Lab Immunol 1999, 6:783-786

78. Lawoko A, Johansson B, Rabinayaran D, Pipkorn R, Blomberg J: Increased immunoglobulin $G$, but not $M$, binding to endogenous retroviral antigens in HIV-1 infected persons. J Med Virol 2000, 62:435-444.

79. Jern P, Sperber GO, Blomberg J: Use of endogenous retroviral sequences (ERVs) and structural markers for retroviral phylogenetic inference and taxonomy. Retrovirology 2005, 2:50.

80. Tandon R, SenGupta D, Ndhlovu LC, Vieira RG, Jones RB, York VA, et al: Identification of human endogenous retrovirus (HERV)-specific T cell responses in vertically HIV-1-infected subjects. J Virol 2011, 85:11526-11531.

81. Schiavetti F, Thonnard J, Colau D, Boon T, Coulie PG: A human endogenous retroviral sequence encoding an antigen recognized on melanoma by cytolytic T lymphocytes. Cancer Res 2002, 62:5510-5516.

82. Takahashi Y, Harashima N, Kajigaya S, Yokoyama H, Cherkasova E, McCoy JP, et al: Regression of human kidney cancer following allogeneic stem cell transplantation is associated with recognition of an HERV-E antigen by $T$ cells. J Clin Invest 2008, 118:1099-1109.

83. NCBI nucleotide database. [http://www.ncbi.n/m.nih.gov/nucleotide/].

84. BioEdit Sequence Alignment Editor version 7.0.9. [http://www.mbio.ncsu. edu/BioEdit/bioedit.html]

85. MEGA 5 software package. [http://www.megasoftware.net/]

86. van der Kuyl AC: Characterization of a full-length endogenous betaretrovirus, EqERV-beta1, in the genome of the horse (Equus caballus). Viruses 2011, 3:620-628

87. Armbruester V, Sauter M, Krautkraemer E, Meese E, Kleiman A, Best B, et al: A novel gene from the human endogenous retrovirus $K$ expressed in transformed cells. Clin Cancer Res 2002, 8:1800-1807.

doi:10.1186/1742-4690-9-6

Cite this article as: van der Kuyl: HIV infection and HERV expression: a review. Retrovirology 2012 9:6.

\section{Submit your next manuscript to BioMed Central and take full advantage of:}

- Convenient online submission

- Thorough peer review

- No space constraints or color figure charges

- Immediate publication on acceptance

- Inclusion in PubMed, CAS, Scopus and Google Scholar

- Research which is freely available for redistribution

Submit your manuscript at www.biomedcentral.com/submit
Ciomed Central 